International Journal of Pure and Applied Mathematics

Volume 109 No. 2 2016, 153-175

ISSN: 1311-8080 (printed version); ISSN: 1314-3395 (on-line version)

url: http://www.ijpam.eu

doi: 10.12732/ijpam.v109i2.1

\title{
SEVERAL NOTIONS OF GENERALIZED SEMI-COMPACTNESS IN DOUBLE FUZZY TOPOLOGICAL SPACES
}

\author{
Fatimah M. Mohammed ${ }^{1 \S}$, M.S.M. Noorani ${ }^{2}$, A. Ghareeb ${ }^{3}$ \\ ${ }^{1,2}$ School of Mathematical Sciences \\ Universiti Kebangsaan Malaysia \\ 43600 UKM Bangi, Selangor, MALAYSIA \\ ${ }^{1}$ College of Education \\ Tikrit University, IRAQ \\ ${ }^{3}$ Department of Mathematics \\ Faculty of Science \\ South Valley University \\ Qena, EGYPT
}

\begin{abstract}
The purpose of this paper is to introduce the notions of several types of semicompactness in double fuzzy topological spaces known as generalized double fuzzy semicompactness, generalized double fuzzy nearly semi-compactness and generalized double fuzzy weakly semi-compactness. Some interesting properties and characterizations of the new concepts are introduced and studied.
\end{abstract}

AMS Subject Classification: 54A40, 54D30, 03E72

Key Words: double fuzzy topology, generalized double fuzzy semi-compact, generalized double fuzzy nearly semi-compact, generalized double fuzzy weakly semi-compact.

Received: July 5, 2015

Published: August 30, 2016

$\S$ Correspondence author
(C) 2016 Academic Publications, Ltd. url: www.acadpubl.eu 


\section{Introduction}

The concept of fuzzy sets was introduce by Zadeh [1]. Later on, C. L. Chang [2] introduced the concept of fuzzy topology, then the generalizations of the concept of fuzzy topology have been done by many authors. In [3], Atanassove introduced the idea of intuitionistic fuzzy sets, then Coker [4, 5], introduced the concept of intuitionistic fuzzy topological spaces. On the other hand, as a generalization of fuzzy topological spaces Samanta and Mondal [6], introduced the concept of intuitionistic gradation of openness. In 2005, the term" intuitionistic" is ended by Garcia and Rodabaugh [7]. They proved that the term "intuitionistic" is unsuitable in mathematics and applications and they replaced it by "double".

In 2010, [8] was introduce the concept of generalized fuzzy compactness in Ltopological spaces. Later in 2012, several types of $(r, s)$-fuzzy compactness was define by an $(r, s)$-fuzzy regular semiopen sets [9] such as $(r, s)$-Rs-compactness, $(r, s)$-fuzzy almost Rs-compactness and $(r, s)$-weakly Rs-compactness in double fuzzy topological spaces.

In this paper, we aim to introduce new kinds of compactness in double fuzzy topological spaces named generalized double fuzzy semi-compact, generalized double fuzzy nearly semi-compact and generalized double fuzzy weakly semi-compact. Some properties and characterizations of these concepts are investigated by using fuzzy filter bases.

\section{Preliminaries}

Throughout this paper, Let $X$ be a non-empty set, $I$ the unit interval $[0,1]$, $I_{0}=(0,1]$ and $I_{1}=[0,1)$. A fuzzy set $\lambda$ is quasi-coincident with a fuzzy set $\mu$ and defined by $\lambda q \mu$ iff there exists $x \in X$ such that $\lambda(x)+\mu(x)>1$ and they are not quasi-coincident which is denoted by $\lambda \bar{q} \mu$. The family of all fuzzy sets on $X$ is denoted by $I^{X}$. By $\underline{0}$ and $\underline{1}$, we denote the smallest and the greatest fuzzy sets on $\mathrm{X}$. For a fuzzy set $\lambda \in I^{X}, \underline{1}-\lambda$ denotes its complement. Given a function $f: I^{X} \longrightarrow I^{Y}$ and its inverse $f^{-1}: I^{Y} \longrightarrow I^{X}$ are defined by $f(\lambda)(y)=\bigvee_{f(x)=y} \lambda(x)$ and $f^{-1}(\mu)(x)=\mu(f(x))$, for each $\lambda \in I^{X}, \mu \in I^{Y}$ and $x \in X$, respectively. All other notations are standard notations of fuzzy set theory.

Definition 2.1. [6] A double fuzzy topology $\left(\tau, \tau^{*}\right)$ on $X$ is a pair of maps $\tau, \tau^{*}: I^{X} \rightarrow I$, which satisfies the following properties: 
(O1) $\tau(\lambda) \leq \underline{1}-\tau^{*}(\lambda)$ for each $\lambda \in I^{X}$.

(O2) $\tau\left(\lambda_{1} \wedge \lambda_{2}\right) \geq \tau\left(\lambda_{1}\right) \wedge \tau\left(\lambda_{2}\right)$ and $\tau^{*}\left(\lambda_{1} \wedge \lambda_{2}\right) \leq \tau^{*}\left(\lambda_{1}\right) \vee \tau^{*}\left(\lambda_{2}\right)$ for each $\lambda_{1}$, $\lambda_{2} \in I^{X}$.

(O3) $\tau\left(\bigvee_{i \in \Gamma} \lambda_{i}\right) \geq \bigwedge_{i \in \Gamma} \tau\left(\lambda_{i}\right)$ and $\tau^{*}\left(\bigvee_{i \in \Gamma} \lambda_{i}\right) \leq \bigvee_{i \in \Gamma} \tau^{*}\left(\lambda_{i}\right)$ for each $\lambda_{i} \in I^{X}$, $i \in \Gamma$.

The triplet $\left(X, \tau, \tau^{*}\right)$ is called a double fuzzy topological space (dfts, for short). A fuzzy set $\lambda$ is called an $(r, s)$-fuzzy open $((r, s)$-fo, for short) if $\tau(\lambda) \geq r$ and $\tau^{*}(\lambda) \leq s, \lambda$ is called an $(r, s)$-fuzzy closed $((r, s)$-fc, for short) iff $\underline{1}-\lambda$ is an $(r, s)$-fo set. Let $\left(X, \tau_{1}, \tau_{1}^{*}\right)$ and $\left(Y, \tau_{2}, \tau_{2}^{*}\right)$ be two dfts's. A function $f: X \rightarrow Y$ is said to be a double fuzzy continuous iff $\tau_{1}\left(f^{-1}(\nu)\right) \geq \tau_{2}(\nu)$ and $\tau_{1}^{*}\left(f^{-1}(\nu)\right) \leq \tau_{2}^{*}(\nu)$ for each $\nu \in I^{Y}$.

Theorem 2.1. [10] Let $\left(X, \tau, \tau^{*}\right)$ be a dfts. Then double fuzzy closure operator and double fuzzy interior operator of $\lambda \in I^{X}$ are defined by

$$
\begin{gathered}
C_{\tau, \tau^{*}}(\lambda, r, s)=\bigwedge\left\{\mu \in I^{X} \mid \lambda \leq \mu, \tau(\underline{1}-\mu) \geq r, \tau^{*}(\underline{1}-\mu) \leq s\right\}, \\
I_{\tau, \tau^{*}}(\lambda, r, s)=\bigvee\left\{\mu \in I^{X} \mid \mu \leq \lambda, \tau(\mu) \geq r, \tau^{*}(\mu) \leq s\right\} .
\end{gathered}
$$

Where $r \in I_{0}$ and $s \in I_{1}$ such that $r+s \leq 1$.

Definition 2.2. Let $\left(X, \tau, \tau^{*}\right)$ be a dfts. For each $\lambda, \mu \in I^{X}, r \in I_{0}$ and $s \in I_{1}$.

(1) an $(r, s)$-fuzzy semiopen $[11,12]$ (for short, $(r, s)$-fso) if

$$
\lambda \leq C_{\tau, \tau^{*}}\left(I_{\tau, \tau^{*}}(\lambda, r, s), r, s\right) .
$$

A fuzzy set $\lambda$ is called $(r, s)$-fuzzy semiclosed (for short, $(r, s)$-fsc) if $\underline{1}-\lambda$ is an $(r, s)$-fuzzy semiopen set.

(2) A fuzzy set $\lambda$ is called an $(r, s)$-generalized fuzzy semi closed $[11,12]$ (briefly, $(r, s)$-gfsc) if $C_{\tau, \tau^{*}}(\lambda, r, s) \leq \mu, \lambda \leq \mu$ and $\mu$ is $(r, s)$-fso set. $\lambda$ is called an $(r, s)$-generalized fuzzy semi open (briefly, $(r, s)$-gfso) iff $\underline{1}-\lambda$ is $(r, s)$-gfsc set.

(3) An $(r, s)$-generalized fuzzy semi-closure of $\lambda[11,12]$ is defined by

$$
G S C_{\tau, \tau^{*}}(\lambda, r, s)=\bigwedge\left\{\mu \in I^{X} \mid \lambda \leq \mu\right.
$$

and $\mu$ is $(r, s)$-gfsc $\}$. 
(4) An $(r, s)$-generalized fuzzy semi-interior of $\lambda[13]$ is defined by

$$
G S I_{\tau, \tau^{*}}(\lambda, r, s)=\vee\left\{\mu \in I^{X} \mid \lambda \leq \mu\right.
$$

and $\mu$ is $(r, s)$-gfso $\}$.

Definition 2.3. [14] Let $\left(X, \tau_{1}, \tau_{1}^{*}\right)$ and $\left(Y, \tau_{2}, \tau_{2}^{*}\right)$ be dfts's. A function $f:\left(X, \tau_{1}, \tau_{1}^{*}\right) \rightarrow\left(Y, \tau_{2}, \tau_{2}^{*}\right)$ is said to be generalized double fuzzy semi-irresolute (briefly, gdfs-irr) if $f^{-1}(\mu)$ is $(r, s)$-gfso set for each $(r, s)$-gfso set $\mu \in I^{Y}, r \in I_{0}$ and $s \in I_{1}$.

\section{Generalized Double Fuzzy Semi-Compact Spaces}

Definition 3.1. Let $\left(X, \tau, \tau^{*}\right)$ be a dfts. A fuzzy set $\lambda$ in $I^{X}$ is called an $(r, s)$-generalized fuzzy semi regular-open set (briefly, $(r, s)$-gfsro) iff

$$
G S I_{\tau, \tau^{*}}\left(G S C_{\tau, \tau^{*}}(\lambda, r, s), r, s\right)=\lambda
$$

for each $r \in I_{0}$ and $s \in I_{1}$. $\lambda$ is called an $(r, s)$-generalized fuzzy semi regularclosed (briefly, $(r, s)$-gfsrc) iff $\underline{1}-\lambda$ is $(r, s)$-gfsro set.

Remark 3.1. Let $\left(X, \tau, \tau^{*}\right)$ be a dfts. For each $\lambda \in I^{X}, r \in I_{0}$ and $s \in I_{1}$, the following statements hold:

(1) $G S I_{\tau, \tau^{*}}(\underline{1}-\lambda, r, s)=\underline{1}-G S C_{\tau, \tau^{*}}(\lambda, r, s)$.

(2) $G S C_{\tau, \tau^{*}}(\underline{1}-\lambda, r, s)=\underline{1}-G S I_{\tau, \tau^{*}}(\lambda, r, s)$.

(3) If $\lambda$ is an $(r, s)$-gfsro set then,

$$
G S I_{\tau, \tau^{*}}(\lambda, r, s)=G S I_{\tau, \tau^{*}}\left(G S C_{\tau, \tau^{*}}(\lambda, r, s), r, s\right)
$$

and

$$
G S C_{\tau, \tau^{*}}(\lambda, r, s)=G S C_{\tau, \tau^{*}}\left(G S I_{\tau, \tau^{*}}(\lambda, r, s), r, s\right)
$$

Theorem 3.1. Let $\left(X, \tau, \tau^{*}\right)$ be a dfts $\lambda \in I^{X}, r \in I_{0}$ and $s \in I_{1} . \lambda$ is an $(r, s)$-gfsrc set if and only if $G S C_{\tau, \tau^{*}}\left(G S I_{\tau, \tau^{*}}(\lambda, r, s), r, s\right)=\lambda$. 
Proof. Suppose that $\lambda$ is an $(r, s)$-gfsrc set $r \in I_{0}$ and $s \in I_{1}$, so by the definition, $\underline{1}-\lambda$ is an $(r, s)$-gfsro set in $I^{X}$. Then

$$
G S I_{\tau, \tau^{*}}\left(G S C_{\tau, \tau^{*}}(\underline{1}-\lambda, r, s), r, s\right)=\underline{1}-\lambda,
$$

so

$$
\underline{1}-\left(G S I_{\tau, \tau^{*}}\left(G S C_{\tau, \tau^{*}}(\underline{1}-\lambda, r, s), r, s\right)\right)=\underline{1}-(\underline{1}-\lambda) .
$$

$\Rightarrow$

$$
G S C_{\tau, \tau^{*}}\left(G S I_{\tau, \tau^{*}}(\lambda, r, s), r, s\right)=\lambda .
$$

Conversely, suppose that $G S C_{\tau, \tau^{*}}\left(G S I_{\tau, \tau^{*}}(\lambda, r, s), r, s\right)=\lambda$ for each $\lambda \in I^{X}$, $r \in I_{0}$ and $s \in I_{1}$, we have to prove $\lambda$ is an $(r, s)$-gfsrc set, i.e, $\underline{1}-\lambda$ is an $(r, s)$-gfsro set, so by hypothesis

$$
G S C_{\tau, \tau^{*}}\left(G S I_{\tau, \tau^{*}}(\underline{1}-\lambda, r, s), r, s\right)=\underline{1}-\lambda .
$$

Therefore,

$$
\underline{1}-\left(G S C_{\tau, \tau^{*}}\left(G S I_{\tau, \tau^{*}}(\underline{1}-\lambda, r, s), r, s\right)\right)=\underline{1}-(\underline{1}-\lambda)
$$

$\Rightarrow$

$$
G S I_{\tau, \tau^{*}}\left(G S C_{\tau, \tau^{*}}(\lambda, r, s), r, s\right)=\lambda .
$$

So by the definition we get that $\underline{1}-\lambda$ is an $(r, s)$-gfsrc set.

Definition 3.2. For each $\lambda, \mu \in I^{X}, r \in I_{0}$ and $s \in I_{1}$, a dfts $\left(X, \tau, \tau^{*}\right)$ is said to be:

(1) generalized double fuzzy semi-compact if for each family $\left\{\lambda_{i}: i \in J\right\}$ of an $(r, s)$-gfso sets such that $\bigvee_{i \in J} \lambda_{i}=\underline{1}$, there exist a finite subfamily $J_{0}$ of $J$ such that

$$
\bigvee_{i \in J_{0}} \lambda_{i}=\underline{1}
$$

(2) generalized double fuzzy semi-compact relative to $X$ if for each family $\left\{\lambda_{i}: i \in J\right\}$ of an $(r, s)$-gfo sets of $X$ such that $\bigvee_{i \in J} \lambda_{i}(x) \geq \mu_{i}(x)$ for each $x \in \operatorname{Sup}(\lambda)$, there exists a finite subfamily $J_{0}$ of $J$ such that for each $x \in \operatorname{Sup}(\lambda)$,

$$
\bigvee_{i \in J_{0}} \lambda_{i}(x) \geq \mu_{i}(x)
$$


(3) generalized double fuzzy nearly semi-compact if for each family $\left\{\lambda_{i}: i \in\right.$ $J\}$ of an $(r, s)$-gfsro sets such that $\bigvee_{i \in J}\left(\lambda_{i}\right)=\underline{1}$, there exists a finite subfamily $J_{0}$ of $J$ such that

$$
\bigvee_{i \in J_{0}} \lambda_{i}=\underline{1}
$$

(4) generalized double fuzzy weakly semi-compact if for each family $\left\{\lambda_{i}\right.$ : $i \in J\}$ of an $(r, s)$-gfso sets such that $\bigvee_{i \in J}\left(\lambda_{i}\right)=\underline{1}$, there exists a finite subfamily $J_{0}$ of $J$ such that

$$
G S C_{\tau, \tau^{*}}\left(\bigvee_{i \in J_{0}} \lambda_{i}, r, s\right)=\underline{1}
$$

Remark 3.2. From Definition 3.2, clearly we have the following:

generalized double fuzzy nearly semi-compact

$$
\Downarrow
$$

generalized double fuzzy semi-compact

$\Downarrow$

generalized double fuzzy weakly semi-compact

Definition 3.3. Let $\left(X, \tau_{1}, \tau_{1}^{*}\right)$ and $\left(Y, \tau_{2}, \tau_{2}^{*}\right)$ be dfts's. A function $f$ : $\left(X, \tau_{1}, \tau_{1}^{*}\right) \rightarrow\left(Y, \tau_{2}, \tau_{2}^{*}\right)$ is called generalized double fuzzy semiopen (briefly, gdfs-open) if $f(\lambda)$ is an $(r, s)$-gfso for each $(r, s)$-gfso set $\lambda \in I^{X}, r \in I_{0}$ and $s \in I_{1}$.

Definition 3.4. Let $\left(X, \tau, \tau^{*}\right)$ be a dfts. A collection $\left\{\lambda_{i}: i \in J\right\}$ of an $(r, s)$-gfso sets in $I^{X}$ is said to be an $(r, s)$-gfso cover of a fuzzy set $\lambda \in I^{X}$, $r \in I_{0}$ and $s \in I_{1}$ iff $\bigvee_{i \in J} \lambda_{i}(x)=\underline{1}$, for each $x \in X$.

Proposition 3.1. Let $\left(X, \tau_{1}, \tau_{1}^{*}\right)$ and $\left(Y, \tau_{2}, \tau_{2}^{*}\right)$ be dfts's. If a function $f:\left(X, \tau_{1}, \tau_{1}^{*}\right) \rightarrow\left(Y, \tau_{2}, \tau_{2}^{*}\right)$ is a surjective gdfs-open and $\left(Y, \tau_{2}, \tau_{2}^{*}\right)$ is generalized double fuzzy semi-compact, then $\left(X, \tau_{1}, \tau_{1}^{*}\right)$ is a generalized double fuzzy semicompact. 
Proof. Suppose $\left\{\lambda_{i}: i \in J\right\}$ is an $(r, s)$-gfso cover of $\mathrm{X}, r \in I_{0}$ and $s \in I_{1}$. Then, $\left\{f\left(\lambda_{i}\right): i \in J\right\}$ is an $(r, s)$-gfso cover of Y. But $\left(Y, \tau_{2}, \tau_{2}^{*}\right)$ is generalized double fuzzy semi-compact, then there exist a finite subfamily $J_{0}$ of $J$ and $\left\{f\left(\lambda_{i}\right): i \in J_{0}\right\}$ such that

$$
\bigvee_{i \in J_{0}}\left(f\left(\lambda_{i}\right)\right)=\underline{1}
$$

But

$$
\bigvee_{i \in J_{0}}\left(f\left(\lambda_{i}\right)\right)=f\left(\bigvee_{i \in J_{0}} \lambda_{i}\right)=\underline{1}
$$

and

$$
\underline{1}=f^{-1}(\underline{1})=f^{-1}\left(f\left(\bigvee_{i \in J_{0}} \lambda_{i}\right)\right)=\bigvee_{i \in J_{0}} \lambda_{i},
$$

Hence, $\left(X, \tau_{1}, \tau_{1}^{*}\right)$ is a generalized double fuzzy semi-compact.

Proposition 3.2. A dfts $\left(X, \tau, \tau^{*}\right)$ is generalized double fuzzy semicompact iff for each collection $\left\{\lambda_{i}: i \in J\right\}$ of an $(r, s)$-gfsc sets of $X, r \in I_{0}$ and $s \in I_{1}$, then $\bigwedge_{i \in J} \lambda_{i} \neq \underline{0}$.

Proof. Suppose that $\left\{\lambda_{i}: i \in J\right\}$ is a family of an $(r, s)$-gfsc sets in a generalized double fuzzy semi-compact $\left(X, \tau, \tau^{*}\right), r \in I_{0}$ and $s \in I_{1}$, such that

$$
\bigwedge_{i \in J} \lambda_{i}=\underline{0} .
$$

Then,

$$
\bigvee_{i \in J}\left(\underline{1}-\lambda_{i}\right)=\underline{1}
$$

But $\left\{\underline{1}-\lambda_{i}: i \in J\right\}$ is a collection of an $(r, s)$-gfso sets and a $\operatorname{dfts}\left(X, \tau, \tau^{*}\right)$ is generalized double fuzzy semi-compact, then there exists a finite subfamily $J_{0}$ of $J$ such that

$$
\bigvee_{i \in J_{0}}\left(\underline{1}-\lambda_{i}\right)=\underline{1}
$$

Therefore,

$$
\bigwedge_{i \in J_{0}} \lambda_{i}=\underline{0}
$$

which is a contradiction. Hence

$$
\bigwedge_{i \in J} \lambda_{i} \neq \underline{0}
$$


Conversely, suppose that $\left\{\lambda_{i}: i \in J\right\}$ is a family of an $(r, s)$-gfso sets covers $\left(X, \tau, \tau^{*}\right)$. Then, $\bigvee_{i \in J} \lambda_{i}=\underline{1}$. Now, let for each subfamily $J_{0}$ of $J$,

$$
\bigvee_{i \in J} \lambda_{i} \neq \underline{1} \Rightarrow \bigwedge_{i \in J_{0}}\left(\underline{1}-\lambda_{i}\right) \neq \underline{0} .
$$

i.e, $\left\{\underline{1}-\lambda_{i}: i \in J\right\}$ is the family of an $(r, s)$-gfsc sets satisfies the finite intersection property. But,

$$
\bigwedge_{i \in J}\left(\underline{1}-\lambda_{i}\right) \neq \underline{0} \Rightarrow \bigvee_{i \in J} \lambda_{i} \neq \underline{1} .
$$

which is a contradiction. Hence $\left(X, \tau, \tau^{*}\right)$ is generalized double fuzzy semicompact.

Proposition 3.3. Let $\left(X, \tau_{1}, \tau_{1}^{*}\right)$ and $\left(Y, \tau_{2}, \tau_{2}^{*}\right)$ be dfts's. A function $f:\left(X, \tau_{1}, \tau_{1}^{*}\right) \rightarrow\left(Y, \tau_{2}, \tau_{2}^{*}\right)$ is a gdfs-irr iff

$$
f\left(G S C_{\tau_{1}, \tau_{1}^{*}}(\lambda, r, s)\right) \leq G S C_{\tau_{2}, \tau_{2}^{*}}(f(\lambda), r, s),
$$

for each $\lambda \in I^{X}, r \in I_{0}$ and $s \in I_{1}$.

Proof. Suppose that $\lambda \in I^{X}, r \in I_{0}$ and $s \in I_{1}$. Then, $G S C_{\tau_{2}, \tau_{2}^{*}}(f(\lambda), r, s)$ is an $(r, s)$-gfsc set in $I^{Y}$. But $f$ is a a gdfs-irr function and

$$
f^{-1}\left(G S C_{\tau_{2}, \tau_{2}^{*}}(f(\lambda), r, s)\right)
$$

is an $(r, s)$-gfsc set in $I^{X}$. Therefore,

$$
G S C_{\tau_{1}, \tau_{1}^{*}}\left(f^{-1}\left(G S C_{\tau_{2}, \tau_{2}^{*}}(f(\lambda), r, s)\right), r, s\right)=f^{-1}\left(G S C_{\tau_{2}, \tau_{2}^{*}}(f(\lambda), r, s)\right) .
$$

Since

$$
\begin{gathered}
\lambda \leq f^{-1}(f(\lambda)) \\
G S C_{\tau_{1}, \tau_{1}^{*}}(\lambda, r, s) \leq G S C_{\tau_{1}, \tau_{1}^{*}}\left(f^{-1}(f(\lambda)), r, s\right) \\
\leq G S C_{\tau_{1}, \tau_{1}^{*}}\left(f^{-1}\left(G S C_{\tau_{2}, \tau_{2}^{*}}(f(\lambda), r, s)\right), r, s\right) \\
=f^{-1}\left(G S C_{\tau_{2}, \tau_{2}^{*}}(f(\lambda), r, s)\right) .
\end{gathered}
$$

i.e, $f\left(G S C_{\tau_{1}, \tau_{1}^{*}}(\lambda, r, s)\right) \leq G S C_{\tau_{2}, \tau_{2}^{*}}(f(\lambda), r, s)$. 
Conversely, suppose that $\lambda$ is an $(r, s)$-gfsc set in $I^{Y}, r \in I_{0}$ and $s \in I_{1}$. Then,

$$
G S C_{\tau_{1}, \tau_{1}^{*}}(\lambda, r, s)=\lambda
$$

But,

$$
\begin{aligned}
\left.G S C_{\tau_{2}, \tau_{2}^{*}}\left(f^{-1}(\lambda), r, s\right)\right) & \leq f^{-1}\left(G S C_{\tau_{1}, \tau_{1}^{*}}\left(f\left(f^{-1}(\lambda)\right), r, s\right)\right) \\
& =f^{-1}\left(G S C_{\tau_{1}, \tau_{1}^{*}}(\lambda, r, s)\right) \\
& =f^{-1}(\lambda) .
\end{aligned}
$$

Also,

$$
f^{-1}(\lambda) \leq G S C_{\tau_{1}, \tau_{1}^{*}}\left(f^{-1}(\lambda), r, s\right)
$$

Thus,

$$
f^{-1}(\lambda)=G S C_{\tau_{1}, \tau_{1}^{*}}\left(f^{-1}(\lambda), r, s\right) .
$$

i.e, $f^{-1}(\lambda)$ is an $(r, s)$-gfsc set in $I^{X}$. Hence, $f$ is gdfs-irr.

Theorem 3.2. Let $\left(X, \tau_{1}, \tau_{1}^{*}\right)$ and $\left(Y, \tau_{2}, \tau_{2}^{*}\right)$ be dfts's. If a function $f:\left(X, \tau_{1}, \tau_{1}^{*}\right) \rightarrow\left(Y, \tau_{2}, \tau_{2}^{*}\right)$ is a gdfs-irr and $\lambda$ is generalized double fuzzy semicompact relative to $\left(X, \tau_{1}, \tau_{1}^{*}\right)$, then so is $f(\lambda)$.

Proof. Suppose that $\left\{\lambda_{i}: i \in J\right\}$ is an $(r, s)$-gfso cover, $r \in I_{0}$ and $s \in I_{1}$, such that for each $f(x) \in Y$ and $x \in X$,

$$
\bigvee_{i \in J}\left(\lambda_{i}\right)(f(x)) \geq f\left(\lambda_{i}\right)(f(x)) .
$$

But $f$ is a gdfs-irr, $\dot{J}=\left\{f^{-1}\left(\lambda_{i}\right): \lambda_{i} \in J\right\}$ is an $(r, s)$-gfso cover, $r \in I_{0}$ and $s \in I_{1}$, so for each $x \in X$ we have

$$
\bigvee_{i \in J}\left(f^{-1}\left(\lambda_{i}\right)(x)\right) \geq \lambda_{i}(x) .
$$

Also, $\lambda_{i}$ is generalized double fuzzy semi-compact relative to $\left(X, \tau_{1}, \tau_{1}^{*}\right)$, then there exists a finite subfamily $J_{0}$ of $J^{\prime}$ such that

$$
\bigvee_{i \in J_{0}}\left(f^{-1}\left(\lambda_{i}\right)\right)(x) \geq \lambda_{i}(x)
$$

for each $x \in X$.

But,

$$
\left.\bigvee_{i \in J_{0}}\left(f^{-1}\left(\lambda_{i}\right)\right)(x)=f^{-1}\left(\bigvee_{i \in J_{0}}\left(\lambda_{i}\right)\right)(x) \geq \lambda_{i}(x)\right)
$$


Therefore,

$$
\bigvee_{i \in J_{0}}\left(\lambda_{i}\right)(x) \geq f\left(\lambda_{i}\right)(x)
$$

That is $f\left(\lambda_{i}\right)$ is generalized double fuzzy semi-compact relative to $\left(Y, \tau_{2}, \tau_{2}^{*}\right)$.

Definition 3.5. A collection of a fuzzy subsets $J$ of a $\operatorname{dfts}\left(X, \tau, \tau^{*}\right)$ is said to be a fuzzy filterbases in $X$ if for each finite subcollection $J_{0}$ of $J$,

$$
\bigwedge_{i \in J_{0}} \lambda_{i} \neq \underline{0}
$$

Theorem 3.3. A dfts $\left(X, \tau, \tau^{*}\right)$ is generalized double fuzzy semi-compact iff for each fuzzy filterbases $J$ of $I^{X}$, the collection

$$
\bigwedge_{i \in J} G S C_{\tau, \tau^{*}}\left(\lambda_{i}, r, s\right) \neq \underline{0} .
$$

Proof. Let $J$ be a fuzzy filterbases of a $\operatorname{dfts}\left(X, \tau, \tau^{*}\right)$ such that

$$
\bigwedge_{i \in J} G S C_{\tau, \tau^{*}}\left(\lambda_{i}, r, s\right) \neq \underline{0}
$$

and let $J$ be an $(r, s)$-gfso cover of $X, r \in I_{0}$ and $s \in I_{1}$ such that $J$ has no finite subcover. So, for each finite subcollection $\left\{\lambda_{i}: \lambda_{i} \leq J\right\}$, there exists $x \in X$ such that $\lambda_{i}(x)<\underline{1}$, for each $\left\{\lambda_{i}: i \in J\right\}$ Then,

$$
\left(\underline{1}-\lambda_{i}\right)(x)>\underline{0} \Rightarrow \bigwedge_{i \in J}\left(\underline{1}-\lambda_{i}\right)(x) \neq \underline{0} .
$$

i.e, $\left\{\left(\underline{1}-\lambda_{i}\right)(x): \lambda_{i} \in J\right\}$ is a fuzzy filterbases in $I^{X}$. But, $J$ is an $(r, s)$-gfso cover of $X$,

$$
\bigvee_{i \in J}\left(\lambda_{i}\right)=\underline{1}
$$

for each $x \in X$ and

$$
\bigwedge_{i \in J}\left(G S C_{\tau, \tau^{*}}\left(\underline{1}-\lambda_{i}\right), r, s\right)(x)=\bigwedge_{i \in J}\left(\underline{1}-\lambda_{i}\right)(x)=\underline{0},
$$

which is a contradiction. Therefore, for each $(r, s)$-gfso cover of $X$ has a finite subcover. Hence, $\left(X, \tau, \tau^{*}\right)$ is generalized double fuzzy semi-compact. 
Conversely, suppose that $\left(X, \tau, \tau^{*}\right)$ is generalized double fuzzy semi-compact and for each fuzzy filterbases $J \in I^{X}, r \in I_{0}$ and $s \in I_{1}$,

$$
\bigwedge_{i \in J} G C_{\tau, \tau^{*}}\left(\lambda_{i}, r, s\right)=\underline{0}
$$

Then,

$$
\bigvee_{i \in J}\left(\underline{1}-\left(G S C_{\tau, \tau^{*}}\left(\lambda_{i}, r, s\right)\right)\right)=\underline{1} .
$$

and hence, $F=\left\{\underline{1}-\left(G S C_{\tau, \tau^{*}}\left(\lambda_{i}, r, s\right)\right): \lambda_{i} \in J\right\}$ is an $(r, s)$-gfso cover of $X$. Therefore,

$$
\bigvee_{i \in J}\left(\underline{1}-\left(G S C_{\tau, \tau^{*}}\left(\lambda_{i}, r, s\right)\right)\right)=\underline{1} \Rightarrow \bigvee_{i \in J}\left(\underline{1}-\lambda_{i}\right)=\underline{1} .
$$

Therefore, $\bigwedge_{i \in J} \lambda_{i}=\underline{0}$. which is a contradiction. Therefore, for each double fuzzy filterbases $J \in I^{\bar{X}}$,

$$
\bigwedge_{i \in J} G S C_{\tau, \tau^{*}}\left(\lambda_{i}, r, s\right) \neq \underline{0} .
$$

Theorem 3.4. A fuzzy set $\lambda$ in $\operatorname{dfts}\left(X, \tau, \tau^{*}\right)$ is generalized double fuzzy semi-compact relative to $\left(X, \tau, \tau^{*}\right)$ iff for each double filterbases $\Gamma$ such that each finite member of $\Gamma$ is quasi conincident with $\lambda, \bigwedge_{i \in \Gamma}\left(G S C_{\tau, \tau^{*}}\left(\mu_{i}, r, s\right)\right) \wedge \lambda \neq \underline{0}$.

Proof. Suppose $\lambda \in I^{X}$ which is not generalized double fuzzy semi-compact relative to $\left(X, \tau, \tau^{*}\right)$. Then, there exists an $(r, s)$-gfso cover $J$ of $\lambda, r \in I_{0}$ and $s \in I_{1}$ such that $J$ has no finite subcover $F$,

$$
\bigvee_{i \in F} \mu_{i}(x) \geq \lambda(x)
$$

for each $x \in X$. Therefore,

$$
\bigvee_{i \in F} \mu_{i}(x)<\lambda(x)
$$

SO

$$
\bigwedge_{i \in F}\left(\underline{1}-\mu_{i}(x)\right)>(\underline{1}-\lambda)(x) \geq \underline{0} .
$$


Hence, $\Gamma=\left\{\left(\underline{1}-\mu_{i}: i \in J\right\}\right.$ is a double fuzzy filterbases and

$$
\bigwedge_{i \in F}\left(\underline{1}-\mu_{i}\right) q \lambda
$$

So, by hypothesis,

$$
\bigwedge_{i \in F}\left(G S C_{\tau, \tau^{*}}\left(\underline{1}-\mu_{i}, r, s\right)\right) \wedge \lambda \neq \underline{0} \Rightarrow \bigwedge_{i \in F}\left(\underline{1}-\mu_{i}\right)(x) \wedge \lambda \neq \underline{0} .
$$

Therefore, for each $x \in X$,

$$
\bigwedge_{i \in J}\left(\underline{1}-\mu_{i}\right)>\underline{0} \Rightarrow \bigvee_{i \in J}\left(\mu_{i}(x)\right)<\underline{1}
$$

which is a contradiction. Hence, $\lambda$ is generalized double fuzzy semi-compact relative to $\left(X, \tau, \tau^{*}\right)$.

Conversely, let $\Gamma$ be a fuzzy filterbases such that each finite member of $\Gamma$ is quasi coincident with $\lambda$ and

$$
\bigwedge_{i \in \Gamma}\left(G S C_{\tau, \tau^{*}}\left(\mu_{i}, r, s\right)\right) \wedge \lambda=\underline{0} .
$$

Then, for each $x \in X$,

$$
\bigwedge_{i \in \Gamma} G S C_{\tau, \tau^{*}}\left(\mu_{i}, r, s\right)(x)=\underline{0} \Rightarrow \bigvee_{i \in \Gamma}\left(\underline{1}-G S C_{\tau, \tau^{*}}\left(\mu_{i}, r, s\right)\right)(x)=\underline{1} .
$$

Since $\lambda$ is generalized double fuzzy semi-compact relative to $\left(X, \tau, \tau^{*}\right)$, so there exists a finite subcover $F$ of $J$ where $J=\left\{\underline{1}-\left(G S C_{\tau, \tau^{*}}\left(\mu_{i}, r, s\right)\right): i \in \Gamma\right\}$ is an $(r, s)$-gfso cover of $\lambda, r \in I_{0}$ and $s \in I_{1}$ such that for each $x \in X$,

$$
\begin{aligned}
& \bigvee_{i \in F}\left(\underline{1}-\left(G S C_{\tau, \tau^{*}}\left(\mu_{i}, r, s\right)\right)(x) \geq \lambda(x) .\right. \\
& \bigwedge_{i \in F}\left(G S C_{\tau, \tau^{*}}\left(\mu_{i}, r, s\right)(x) \leq(\underline{1}-\lambda)(x),\right.
\end{aligned}
$$

so that

$$
\bigwedge_{i \in F}\left(G S C_{\tau, \tau^{*}}\left(\mu_{i}, r, s\right)\right)(x) \bar{q} \lambda
$$

which is contradiction. Therefore, for each fuzzy filterbases $\Gamma$ such that each finite members of $\Gamma$ is quasi coincident with $\lambda$,

$$
\left(\bigwedge_{i \in \Gamma}\left(G S C_{\tau, \tau^{*}}\left(\mu_{i}, r, s\right)\right)(x) \wedge \lambda \neq \underline{0} .\right.
$$


Proposition 3.4. If a dfts $\left(X, \tau, \tau^{*}\right)$ is generalized double fuzzy semicompact space, then each $(r, s)$-gfsc set in $\left(X, \tau, \tau^{*}\right)$ is generalized double fuzzy semi-compact relative to $\left(X, \tau, \tau^{*}\right)$.

Proof. Suppose $\lambda$ is an $(r, s)$-gfsc set in $I^{X}$ and $J$ is any fuzzy filterbases such that $\bigwedge_{i \in J}\left(\mu_{i}\right) q \lambda$ for each $F$ of $J$.

Suppose $J^{*}=\lambda \vee J$ and for each subfamily $\left\{F^{*}: F^{*}\right.$ of $\left.J^{*}\right\}, \bigwedge_{i \in F^{*}-\lambda}\left(\mu_{i}\right) q \lambda$ and $\bigwedge_{\delta \in F^{*}} \delta \neq \underline{0}$. Hence, $J^{*}$ is a fuzzy filterbases in $I^{X}$. But $\left(X, \tau, \tau^{*}\right)$ is generalized double fuzzy semi-compact space, then by Theorem 3.2,

$$
\bigwedge_{i \in J^{*}}\left(G S C_{\tau, \tau^{*}}\left(\mu_{i}, r, s\right)\right) \neq \underline{0} \Rightarrow \bigwedge_{i \in J^{*}} G S C_{\tau, \tau^{*}}\left(\mu_{i}, r, s\right) \bigwedge \lambda \neq \underline{0} .
$$

Hence by Theorem 3.3, $\lambda$ is generalized double fuzzy semi-compact relative to $\left(X, \tau, \tau^{*}\right)$.

Proposition 3.5. For each $\operatorname{dfts}\left(X, \tau, \tau^{*}\right)$, the following conditions are equivalent:

(1) $\left(X, \tau, \tau^{*}\right)$ is generalized double fuzzy nearly semi-compact.

(2) For each family $\left\{\lambda_{i}: i \in J\right\}$ of an $(r, s)$-gfsro sets such that $\bigwedge_{i \in J_{0}} \lambda_{i} \neq \underline{0}$, where $J_{0}$ of $J$ is any finite subfamily, $\bigwedge_{i \in J} \lambda_{i} \neq \underline{0}$.

Proof. $(1) \Rightarrow(2)$ Suppose $\left\{\lambda_{i}: i \in J\right\}$ is a family of an $(r, s)$-gfsro sets such that

$$
\bigwedge_{i \in J_{0}} \lambda_{i} \neq \underline{0}
$$

for each finite subfamily $J_{0}$ of $J$.

Now, suppose that

$$
\bigwedge_{i \in J} \lambda_{i}=\underline{0} .
$$

Then,

$$
\underline{1}-\bigwedge_{i \in J} \lambda_{i}=\underline{1}
$$

But,

$$
\underline{1}-\bigwedge_{i \in J} \lambda_{i}=\bigvee_{i \in J}\left(\underline{1}-\lambda_{i}\right)
$$

then by (1), there exists a finite subfamily $J_{0}$ of $J$ such that

$$
\bigvee_{i \in J_{0}}\left(\underline{1}-\lambda_{i}\right)=\underline{1}
$$


So,

$$
\bigwedge_{i \in J_{0}} \lambda_{i}=\underline{0}
$$

which is contradiction. Therefore,

$$
\bigwedge_{i \in J} \lambda_{i} \neq \underline{0}
$$

$(2) \Rightarrow(1)$ Suppose $\left\{\lambda_{i}: i \in J\right\}$ is a family of an $(r, s)$-gfsro sets such that $\bigvee_{i \in J} \lambda_{i}=\underline{1}$. Then, $\left\{\left(\underline{1}-\lambda_{i}\right): \lambda_{i} \in J\right\}$ is a family of an $(r, s)$-gfsrc sets. Now, for each subfamily $J_{0}$ of $J$, let

$$
\bigvee_{i \in J_{0}} \lambda_{i}=\underline{1} \Rightarrow \bigwedge_{i \in J_{0}}\left(\underline{1}-\lambda_{i}\right)=\underline{0} .
$$

By (2),

$$
\bigwedge_{i \in J}\left(\underline{1}-\lambda_{i}\right) \neq \underline{0} \Rightarrow \bigvee_{i \in J} \lambda_{i} \neq \underline{1},
$$

which is a contradiction. Hence, $\left(X, \tau, \tau^{*}\right)$ is generalized double fuzzy nearly semi-compact.

Theorem 3.5. A dfts $\left(X, \tau, \tau^{*}\right)$ is a generalized double fuzzy weakly semi-compact iff for each family $\left\{\lambda_{i}: i \in J\right\}$ of an $(r, s)$-gfsc sets such that $\bigwedge_{i \in J} \lambda_{i}=\underline{0}$, there exists a finite subfamily $J_{0}$ of $J$ such that

$$
G S I_{\tau, \tau^{*}}\left(\bigwedge_{i \in J_{0}} \lambda_{i}, r, s\right)=\underline{0} .
$$

Proof. Suppose $\left(X, \tau, \tau^{*}\right)$ is a generalized double fuzzy weakly semi-compact and $\left\{\lambda_{i}: i \in J\right\}$ is a family of $(r, s)$-gfsc sets such that

$$
\bigwedge_{i \in J} \lambda_{i}=\underline{0} \text {. }
$$

Then,

$$
\underline{1}-\bigwedge_{i \in J} \lambda_{i}=\underline{1} \Rightarrow \bigvee_{i \in J}\left(\underline{1}-\lambda_{i}\right)=\underline{1} .
$$

But, $\left(X, \tau, \tau^{*}\right)$ is a generalized double fuzzy weakly semi-compact then, there exists a finite subfamily $J_{0}$ of $J$ such that

$$
G S C_{\tau, \tau^{*}}\left(\bigvee_{i \in J_{0}}\left(\underline{1}-\lambda_{i}\right), r, s\right)=\underline{1}
$$


Therefore,

$$
\underline{1}-\left(G S C_{\tau, \tau^{*}}\left(\bigvee_{i \in J_{0}}\left(\underline{1}-\lambda_{i}\right), r, s\right)\right)=\underline{0} \Rightarrow G S I_{\tau, \tau^{*}}\left(\underline{1}-\bigvee_{i \in J_{0}}\left(\underline{1}-\lambda_{i}\right), r, s\right)=\underline{0} .
$$

i.e, $G S I_{\tau, \tau^{*}}\left(\bigwedge_{i \in J_{0}}\left(\lambda_{i}, r, s\right)\right)=\underline{0}$. Then,

Conversely, Suppose $J$ is a family of an $(r, s)$-gfso sets such that $\bigvee_{i \in J} \mu_{i}=\underline{1}$.

$$
\underline{1}-\bigvee_{i \in J} \mu_{i}=\underline{0} \Rightarrow \bigwedge_{i \in J}\left(\underline{1}-\mu_{i}\right)=\underline{0} .
$$

Then, there exists a finite subfamily $J_{0}$ of $J$ such that $G S I_{\tau, \tau^{*}}\left(\bigwedge_{i \in J_{0}}(\underline{1}-\right.$ $\left.\left.\mu_{i}\right), r, s\right)=\underline{0}$. Then,

$$
\underline{1}-\left(G S I_{\tau, \tau^{*}}\left(\bigwedge_{i \in J_{0}}\left(\underline{1}-\mu_{i}\right), r, s\right)\right)=\underline{1} \Rightarrow G S C_{\tau, \tau^{*}}\left(\underline{1}-\bigwedge_{i \in J_{0}}\left(\underline{1}-\mu_{i}\right), r, s\right)=\underline{1} .
$$

Therefore,

$$
G S C_{\tau, \tau^{*}}\left(\bigvee_{i \in J_{0}} \mu_{i}, r, s\right)=\underline{1}
$$

and hence, $\left(X, \tau, \tau^{*}\right)$ is a generalized double fuzzy weakly semi-compact space.

Proposition 3.6. Let $\left(X, \tau, \tau^{*}\right)$ be a dfts. Then

(1) Every $(r, s)$-gfsro set is $(r, s)$-gfso.

(2) Every $(r, s)$-gfsrc set is $(r, s)$-gfsc.

(3) The $(r, s)$-generalized fuzzy semi-closure of an $(r, s)$-gfso set is an $(r, s)$ gfsrc.

(4) The $(r, s)$-generalized fuzzy semi-interior of an $(r, s)$-gfsc set is an $(r, s)$ gfsro.

Proof. (1) Suppose $\lambda$ is any $(r, s)$-gfsro set in $I^{X}, r \in I_{0}$ and $s \in I_{1}$. Then,

$$
G S I_{\tau, \tau^{*}}\left(G S C_{\tau, \tau^{*}}(\lambda, r, s), r, s\right)=\lambda
$$

Now,

$$
\begin{aligned}
G S I_{\tau, \tau^{*}}(\lambda, r, s) & =G S I_{\tau, \tau^{*}}\left(G S I_{\tau, \tau^{*}}\left(G S C_{\tau, \tau^{*}}(\lambda, r, s), r, s\right), r, s\right) \\
& =G S I_{\tau, \tau^{*}}\left(G S C_{\tau, \tau^{*}}(\lambda, r, s)\right)
\end{aligned}
$$




$$
=\lambda \text {. }
$$

Therefore, $\lambda$ is an $(r, s)$-gfso set in $I^{X}$.

(2) Suppose $\lambda$ is any $(r, s)$-gfsro set in $I^{X}, r \in I_{0}$ and $s \in I_{1}$. Then,

$$
G S C_{\tau, \tau^{*}}\left(G S I_{\tau, \tau^{*}}(\lambda, r, s), r, s\right)=\lambda .
$$

Now,

$$
\begin{aligned}
G S C_{\tau, \tau^{*}}(\lambda, r, s) & =G S C_{\tau, \tau^{*}}\left(G S C_{\tau, \tau^{*}}\left(G S I_{\tau, \tau^{*}}(\lambda, r, s), r, s\right), r, s\right) \\
& =G S C_{\tau, \tau^{*}}\left(G S I_{\tau, \tau^{*}}(\lambda, r, s)\right) \\
& =\lambda
\end{aligned}
$$

Therefore, $\lambda$ is an $(r, s)$-gfsc set in $I^{X}$.

(3) Suppose $\lambda$ is any $(r, s)$-gfso set in $I^{X}, r \in I_{0}$ and $s \in I_{1}$. Since $\lambda \leq$ $G S C_{\tau, \tau^{*}}(\lambda, r, s)$,

$$
G S I_{\tau, \tau^{*}}(\lambda, r, s) \leq G S I_{\tau, \tau^{*}}\left(G S C_{\tau, \tau^{*}}(\lambda, r, s), r, s\right) .
$$

Also, since $\lambda$ is any $(r, s)$-gfso set in $I^{X}$ and $\lambda \leq G S I_{\tau, \tau^{*}}\left(G S C_{\tau, \tau^{*}}(\lambda, r, s), r, s\right)$. Therefore,

$$
G S C_{\tau, \tau^{*}}(\lambda, r, s) \leq G S C_{\tau, \tau^{*}}\left(G S I_{\tau, \tau^{*}}\left(G S C_{\tau, \tau^{*}}(\lambda, r, s), r, s\right), r, s\right) .
$$

Now,

$$
\begin{aligned}
G S I_{\tau, \tau^{*}}\left(G S C_{\tau, \tau^{*}}(\lambda, r, s), r, s\right) & \leq G S C_{\tau, \tau^{*}}(\lambda, r, s) \\
& \Rightarrow \\
G S C_{\tau, \tau^{*}}\left(G S I_{\tau, \tau^{*}}\left(G S C_{\tau, \tau^{*}}(\lambda, r, s), r, s\right), r, s\right) & \leq G S C_{\tau, \tau^{*}}(\lambda, r, s) .
\end{aligned}
$$

i.e,

$$
G S C_{\tau, \tau^{*}}(\lambda, r, s)=G S C_{\tau, \tau^{*}}\left(G S I_{\tau, \tau^{*}}\left(G S C_{\tau, \tau^{*}}(\lambda, r, s), r, s\right), r, s\right) .
$$

Hence, $\lambda$ is an $(r, s)$-gfso set is an $(r, s)$-gfsrc in $I^{X}$.

(4) Suppose $\lambda$ is any $(r, s)$-gfsc set in $I^{X}, r \in I_{0}$ and $s \in I_{1}$. Since $G S I_{\tau, \tau^{*}}(\lambda, r, s) \leq \lambda$

$$
G S C_{\tau, \tau^{*}}\left(G S I_{\tau, \tau^{*}}(\lambda, r, s), r, s\right) \leq G S C_{\tau, \tau^{*}}(\lambda, r, s) .
$$

Also, since $\lambda$ is any $(r, s)$-gfsc set in $I^{X}$ and $G S C_{\tau, \tau^{*}}\left(G S I_{\tau, \tau^{*}}(\lambda, r, s), r, s\right) \leq \lambda$. Therefore,

$$
G S I_{\tau, \tau^{*}}\left(G S C_{\tau, \tau^{*}}\left(G S I_{\tau, \tau^{*}}(\lambda, r, s), r, s\right), r, s\right) \leq G S I_{\tau, \tau^{*}}(\lambda, r, s) .
$$


Now,

$$
\begin{aligned}
G S I_{\tau, \tau^{*}}(\lambda, r, s) & \leq G S C_{\tau, \tau^{*}}\left(G S I_{\tau, \tau^{*}}(\lambda, r, s), r, s\right) \\
& \Rightarrow G S I_{\tau, \tau^{*}}(\lambda, r, s) \\
& \leq G S I_{\tau, \tau^{*}}\left(G S C_{\tau, \tau^{*}}\left(G S I_{\tau, \tau^{*}}(\lambda, r, s), r, s\right), r, s\right) .
\end{aligned}
$$

i.e,

$$
G S I_{\tau, \tau^{*}}(\lambda, r, s)=G S I_{\tau, \tau^{*}}\left(G S C_{\tau, \tau^{*}}\left(G S I_{\tau, \tau^{*}}(\lambda, r, s), r, s\right), r, s\right) .
$$

Hence, $\lambda$ is an $(r, s)$-gfso set is an $(r, s)$-gfsro in $I^{X}$.

Theorem 3.6. For each a dfts $\left(X, \tau, \tau^{*}\right)$, the following conditions are equivalent:

(1) $\left(X, \tau, \tau^{*}\right)$ is generalized double fuzzy weakly semi-compact.

(2) For each an $(r, s)$-gfsro cover $\left\{\lambda_{i}: i \in J\right\}$ of $X$, there exists a finite subcover $J_{0}$ of $J$ such that

$$
G S C_{\tau, \tau^{*}}\left(\bigvee_{i \in J_{0}} \lambda_{i}, r, s\right)=\underline{1}
$$

(3) For each family $\left\{\lambda_{i}: i \in J\right\}$ of an $(r, s)$-gfsrc of $I^{X}$ such that $\bigwedge_{i \in J} \lambda_{i}=\underline{0}$, there exists a finite subfamily $J_{0}$ of $J$ such that

$$
G S I_{\tau, \tau^{*}}\left(\bigwedge_{i \in J_{0}} \lambda_{i}, r, s\right)=\underline{0} .
$$

Proof. $(1) \Rightarrow(2)$ Suppose $\left\{\lambda_{i}: i \in J\right\}$ is an $(r, s)$-gfsro cover of $X$. Then, $\left\{\lambda_{i}: i \in J\right\}$ is an $(r, s)$-gfso cover of $X$. But by $(1),\left(X, \tau, \tau^{*}\right)$ is generalized double fuzzy weakly semi-compact, then there exists a finite subfamily $J_{0}$ of $J$ such that

$$
G S C_{\tau, \tau^{*}}\left(\bigvee_{i \in J_{0}} \lambda_{i}, r, s\right)=\underline{1} .
$$

$(2) \Rightarrow(3)$ Suppose $\left\{\lambda_{i}: i \in J\right\}$ is a family of an $(r, s)$-gfsrc sets of $I^{X}$ such that

$$
\bigwedge_{i \in J} \lambda_{i}=\underline{0}
$$

Then,

$$
\underline{1}-\bigwedge_{i \in J} \lambda_{i}=\underline{1} \Rightarrow \bigvee_{i \in J}\left(\underline{1}-\lambda_{i}\right)=\underline{1}
$$


So, $\underline{1}-\lambda_{i}$ is an $(r, s)$-gfsro and by (2), there exists a finite subcover $J_{0}$ of $J$ such that

$$
G S C_{\tau, \tau^{*}}\left(\bigvee_{i \in J_{0}}\left(\underline{1}-\lambda_{i}\right), r, s\right)=\underline{1}
$$

Therefore,

$$
\begin{aligned}
G S C_{\tau, \tau^{*}}\left(\underline{1}-\bigwedge_{i \in J_{0}} \lambda_{i}, r, s\right) & =\underline{1} \\
\underline{1}-G S I_{\tau, \tau^{*}}\left(\bigwedge_{i \in J_{0}} \lambda_{i}, r, s\right) & =\underline{1} .
\end{aligned}
$$

Hence, $G S I_{\tau, \tau^{*}}\left(\bigwedge_{i \in J_{0}} \lambda_{i}, r, s\right)=\underline{0}$. $\underline{0}$.

$(3) \Rightarrow(1)$ Suppose $\left\{\lambda_{i}: i \in J\right\}$ is an $(r, s)$-gfsro cover of $X$ such that $\bigwedge_{i \in J} \lambda_{i}=$

Take

$$
\dot{J}=\left\{G S I_{\tau, \tau^{*}}\left(G S C_{\tau, \tau^{*}}\left(\lambda_{i}, r, s\right), r, s\right): i \in J\right\}
$$

is an $(r, s)$-gfso cover $\left\{\lambda_{i}: i \in J\right\}$. Then by (3), there exists a finite subcover

$$
\dot{J}_{0}=\left\{G S I_{\tau, \tau^{*}}\left(G S C_{\tau, \tau^{*}}\left(\lambda_{i}, r, s\right), r, s\right): i \in \dot{J}_{0}\right\}
$$

such that

$$
\begin{aligned}
G S C_{\tau, \tau^{*}}\left(\bigvee_{i \in \hat{J}_{0}}\left(G S I_{\tau, \tau^{*}}\left(G S C_{\tau, \tau^{*}}\left(\lambda_{i}, r, s\right), r, s\right), r, s\right)\right. & =G S C_{\tau, \tau^{*}}\left(\bigvee_{i \in \hat{J}_{0}} \lambda_{i}, r, s\right) \\
& =\underline{1}
\end{aligned}
$$

Therefore, $\left(X, \tau, \tau^{*}\right)$ is generalized double fuzzy weakly semi-compact.

Definition 3.6. For each $\lambda, \mu \in I^{X}, r \in I_{0}$ and $s \in I_{1}$, a dfts $\left(X, \tau, \tau^{*}\right)$ is said to be generalized double fuzzy semi-closed iff for each family $\left\{\lambda_{i}: i \in J\right\}$ of an $(r, s)$-gfso sets such that $\bigvee_{i \in J} \lambda_{i}=\underline{1}$, there is a finite subfamily $J_{0}$ of $J$ such that

$$
\bigvee_{i \in J_{0}} G S C_{\tau, \tau^{*}}\left(\lambda_{i}, r, s\right)=\underline{1}
$$

Theorem 3.7. A dfts $\left(X, \tau, \tau^{*}\right)$ is a generalized double fuzzy semi-closed iff for each family $\left\{\lambda_{i}: i \in J\right\}$ of an $(r, s)$-gfsc sets has a finite subfamily $J_{0}$ of $J$ such that $\bigwedge_{i \in J_{0}} G S I_{\tau, \tau^{*}}\left(\lambda_{i}, r, s\right)=\underline{0}$. 
Proof. Suppose $\left(X, \tau, \tau^{*}\right)$ is a generalized double fuzzy weakly semi-compact and $\left\{\lambda_{i}: i \in J\right\}$ is a family of $(r, s)$-gfsc sets such that $\bigwedge_{i \in J} \lambda_{i}=\underline{0}$. Then, $\bigvee_{i \in J}\left(\underline{1}-\lambda_{i}\right)=\underline{1}$. But, $\underline{1}-\lambda_{i}$ is an $(r, s)$-gfso and $\left(X, \tau, \tau^{*}\right)$ is a generalized double fuzzy semi-closed so, there exists a finite subfamily $J_{0}$ of $J$ such that

$$
\bigvee_{i \in J_{0}} G S C_{\tau, \tau^{*}}\left(\underline{1}-\lambda_{i}, r, s\right)=\underline{1} .
$$

Therefore,

$$
\bigvee_{i \in J_{0}}\left(\underline{1}-G S I_{\tau, \tau^{*}}\left(\lambda_{i}, r, s\right)\right)=\underline{1} \Rightarrow \underline{1}-\bigwedge_{i \in J_{0}} G S I_{\tau, \tau^{*}}\left(\lambda_{i}, r, s\right)=\underline{1}
$$

i.e,

$$
\bigwedge_{i \in J_{0}} G S I_{\tau, \tau^{*}}\left(\lambda_{i}, r, s\right)=\underline{0} .
$$

Conversely, suppose $\left\{\lambda_{i}: i \in J\right\}$ is a family of an $(r, s)$-gfso sets such that

$$
\bigvee_{i \in J} \lambda_{i}=\underline{1} \Rightarrow \bigwedge_{i \in J}\left(\underline{1}-\lambda_{i}\right)=\underline{0} .
$$

But, $\underline{1}-\lambda_{i}$ is an $(r, s)$-gfsc, then there exists a finite subfamily $J_{0}$ of $J$ such that

$$
\bigwedge_{i \in J_{0}}\left(G S I_{\tau, \tau^{*}}\left(\underline{1}-\lambda_{i}, r, s\right)\right)=\underline{0} .
$$

Then,

$$
\begin{gathered}
\bigwedge_{i \in J_{0}}\left(\underline{1}-\left(G S C_{\tau, \tau^{*}}\left(\lambda_{i}, r, s\right)\right)\right)=\underline{0} \\
\Rightarrow \underline{1}-\bigvee_{i \in J_{0}}\left(G S C_{\tau, \tau^{*}}\left(\lambda_{i}, r, s\right)\right)=\underline{0} .
\end{gathered}
$$

Therefore,

$$
\bigvee_{i \in J_{0}} G S C_{\tau, \tau^{*}}\left(\lambda_{i}, r, s\right)=\underline{1}
$$

Hence, $\left(X, \tau, \tau^{*}\right)$ is a generalized double fuzzy semi-closed space.

Theorem 3.8. For each a dfts $\left(X, \tau, \tau^{*}\right)$, the following conditions are equivalent:

(1) $\left(X, \tau, \tau^{*}\right)$ is generalized double fuzzy semi-closed. 
(2) For each family $\left\{\lambda_{i}: i \in J\right\}$ of an $(r, s)$-gfsro sets of $\left(X, \tau, \tau^{*}\right)$ such that $\bigvee_{i \in J} \lambda_{i}=\underline{1}$, there exists a finite subfamily $J_{0}$ of $J$ such that

$$
\bigvee_{i \in J_{0}} G S C_{\tau, \tau^{*}}\left(\lambda_{i}, r, s\right)=\underline{1}
$$

(3) For each family $\left\{\lambda_{i}: i \in J\right\}$ of an $(r, s)$-gfsrc sets of $I^{X}$ such that $\bigwedge_{i \in J} \lambda_{i}=$ $\underline{0}$, there exists a finite subfamily $J_{0}$ of $J$ such that

$$
\bigwedge_{i \in J_{0}} G S I_{\tau, \tau^{*}}\left(\lambda_{i}, r, s\right)=\underline{0}
$$

Proof. (1) $\Rightarrow(2)$ Suppose $\left\{\lambda_{i}: i \in J\right\}$ is an $(r, s)$-gfsro cover of $X$. Then, $\left\{\lambda_{i}: i \in J\right\}$ is an $(r, s)$-gfso cover of $X$. Since $\left(X, \tau, \tau^{*}\right)$ is generalized double fuzzy semi closed, there exists a finite subfamily $J_{0}$ of $J$ such such $\bigvee_{i \in J_{0}} G S C_{\tau, \tau^{*}}\left(\lambda_{i}, r, s\right)=\underline{1}$.

$(2) \Rightarrow(3)$ Suppose $\left\{\lambda_{i}: i \in J\right\}$ is an $(r, s)$-gfsrc sets of $I^{X}, r \in I_{0}$ and $s \in I_{1}$ such that

$$
\bigwedge_{i \in J} \lambda_{i}=\underline{0} \Rightarrow \bigvee_{i \in J}\left(\underline{1}-\lambda_{i}\right)=\underline{1} .
$$

But, $\underline{1}-\lambda_{i}$ is an $(r, s)$-gfsro set, so for each family $\left\{\lambda_{i}: i \in J\right\}$ of an $(r, s)$-gfsro sets of $I^{X}$ there exists a finite subfamily $J_{0}$ of $J$ such that

$$
\bigvee_{i \in J}\left(\underline{1}-\lambda_{i}\right)=\underline{1}
$$

But,

$$
\begin{aligned}
& \bigvee_{i \in J_{0}} G S C_{\tau, \tau^{*}}\left(\underline{1}-\lambda_{i}, r, s\right) \\
= & \bigvee_{i \in J_{0}}\left(\underline{1}-G S I_{\tau, \tau^{*}}\left(\lambda_{i}, r, s\right)\right) \\
= & \underline{1}-\bigwedge_{i \in J_{0}} G S I_{\tau, \tau^{*}}\left(\lambda_{i}, r, s\right)=\underline{1} .
\end{aligned}
$$

Therefore,

$$
\bigwedge_{i \in J_{0}} G S I_{\tau, \tau^{*}}\left(\lambda_{i}, r, s\right)=\underline{0} .
$$


(3) $\Rightarrow(1)$ Suppose $\left\{\lambda_{i}: i \in J\right\}$ is an $(r, s)$-gfsro sets of $I^{X}, r \in I_{0}$ and $s \in I_{1}$ such that $\bigwedge_{i \in J}\left(\lambda_{i}\right)=\underline{0}$. Now, $\dot{J}=\left\{G S C_{\tau, \tau^{*}}\left(G S I_{\tau, \tau^{*}}\left(\underline{1}-\lambda_{i}, r, s\right), r, s\right): i \in J\right\}$ is an $(r, s)$-gfro cover of $X$. But, by $(3)$, there exists a finite subfamily

$$
\dot{J}_{0}=\left\{G S I_{\tau, \tau^{*}}\left(G S C_{\tau, \tau^{*}}\left(\underline{1}-\lambda_{i}, r, s\right), r, s\right): i \in \dot{J}_{0}\right\}
$$

is a finite subfamily of $\left\{\lambda_{i}: i \in J\right\}$ such that

$$
\begin{gathered}
\bigwedge_{i \in J_{0}}\left(G S I_{\tau, \tau^{*}}\left(G S I_{\tau, \tau^{*}}\left(G S C_{\tau, \tau^{*}}\left(\underline{1}-\lambda_{i}, r, s\right), r, s\right), r, s\right)=\underline{0}\right. \\
\Rightarrow \\
\bigvee_{i \in J_{0}} G S C_{\tau, \tau^{*}}\left(\lambda_{i}, r, s\right)=\underline{1} .
\end{gathered}
$$

Therefore, $\left(X, \tau, \tau^{*}\right)$ is generalized double fuzzy semi closed.

Theorem 3.9. Let $\left(X, \tau_{1}, \tau_{1}^{*}\right)$ and $\left(Y, \tau_{2}, \tau_{2}^{*}\right)$ be dfts's. If a function $f:\left(X, \tau_{1}, \tau_{1}^{*}\right) \rightarrow\left(Y, \tau_{2}, \tau_{2}^{*}\right)$ is a gdfs-irr, a surjective function and $\left(X, \tau_{1}, \tau_{1}^{*}\right)$ is a generalized double fuzzy semi-closed then, so is $\left(Y, \tau_{2}, \tau_{2}^{*}\right)$.

Proof. Suppose that $\left\{\lambda_{i}: i \in J\right\}$ is an $(r, s)$-gfso cover of $Y$ and $f$ is gdfsirr function, than $\left\{f^{-1}\left(\lambda_{i}\right): i \in J\right\}$ is an $(r, s)$-gfso cover of $X, r \in I_{0}$ and $s \in I_{1}$ such that $\left\{\lambda_{i}: i \in J\right\}$ is an $(r, s)$-gfso cover of $Y$, so there exists a finite subfamily $\left\{J_{0}: J_{0} \leq J\right\}$ such that

$$
\bigvee_{i \in J_{0}}\left(G S C_{\tau, \tau^{*}}\left(f^{-1}\left(\lambda_{i}\right), r, s\right)\right)=\underline{1}
$$

But $f$ is surjective function and by Proposition 3.3,

$$
\begin{aligned}
\underline{1}=f(\underline{1}) & =f\left(\bigvee_{i \in J_{0}}\left(G S C_{\tau_{1}, \tau_{1}^{*}}\left(f^{-1}\left(\lambda_{i}\right), r, s\right)\right)\right) \\
& \leq \bigvee_{i \in J_{0}}\left(G S C_{\tau_{2}, \tau_{2}^{*}}\left(f\left(f^{-1}\left(\lambda_{i}\right)\right), r, s\right)\right) \\
& =\bigvee_{i \in J_{0}}\left(G S C_{\tau_{2}, \tau_{2}^{*}}\left(\lambda_{i}, r, s\right)\right) .
\end{aligned}
$$

Hence, $\left(Y, \tau_{2}, \tau_{2}^{*}\right)$ is a generalized double fuzzy semi-closed.

Theorem 3.10. A dfts $\left(X, \tau, \tau^{*}\right)$ is a generalized double fuzzy semi-closed iff for each an $(r, s)$-gfso filterbases $F$ in $I^{X}, \bigwedge_{i \in F}\left(G S C_{\tau, \tau^{*}}\left(\lambda_{i}, r, s\right)\right) \neq \underline{0}$. 
Proof. Let $F$ be $(r, s)$-gfso filterbases in $I^{X}$ such that

$$
\bigwedge_{i \in F}\left(G S C_{\tau, \tau^{*}}\left(\lambda_{i}, r, s\right)\right) \neq \underline{0}
$$

and let $\left\{\lambda_{i}: i \in J\right\}$ be any $(r, s)$-gfso cover of $X$. Then,

$$
\bigvee_{i \in J} \lambda_{i}=\underline{1}
$$

Suppose that there exists a finite subfamily $J_{0}$ of $J$ such that

$$
\bigvee_{i \in J_{0}}\left(G S C_{\tau, \tau^{*}}\left(\lambda_{i}, r, s\right)\right)=\underline{1} \Rightarrow \bigwedge_{i \in J_{0}}\left(\underline{1}-G S C_{\tau, \tau^{*}}\left(\lambda_{i}, r, s\right)\right)=\underline{0}
$$

Thus, $\left\{\left(\underline{1}-G S C_{\tau, \tau^{*}}\left(\lambda_{i}, r, s\right)\right): i \in J\right\}=F$ is an $(r, s)$-gfso filterbases in $I^{X}$, so

$$
\bigwedge_{i \in J}\left(G S C_{\tau, \tau^{*}}\left(\underline{1}-\left(G S C_{\tau, \tau^{*}}\left(\lambda_{i}, r, s\right)\right), r, s\right)=\underline{0} .\right.
$$

i.e, each which an $(r, s)$-gfso cover of $X$ has a finite subfamily $J_{0}$ of $J$ such that

$$
\bigvee_{i \in J_{0}}\left(G S C_{\tau, \tau^{*}}\left(\lambda_{i}, r, s\right)\right)=\underline{1}
$$

which is contradiction. Hence $\left(X, \tau, \tau^{*}\right)$ is a generalized double fuzzy semiclosed.

\section{Conclusion}

In this paper, we have introduced the notions of several types of semi-compactness in double fuzzy topological spaces named by generalized double fuzzy semicompactness, generalized double fuzzy nearly semi-compactness and generalized double fuzzy weakly semi-compactness using fuzzy filter bases. Some interesting properties and characterizations of the concepts introduced are studied. Since double fuzzy topology forms an extension of fuzzy topology and general topology, we think that our results can be applied in modren physics and GIS Problems and also, we hope these investigations will further encourage other researchers to explore the interesting connections between this area of topology and fuzzy set. 


\section{Acknowledgments}

The authors would like to acknowledge the grant:UKM Grant DIP-2014-034 and Ministry of Educa-tion, Malaysia grant FRGS/1/2014/ST06/UKM/01/1 for financial support.

\section{References}

[1] L. A. Zadeh, Fuzzy sets, Information and Control, 8, No. 3 (1965), 338-353.

[2] C. L. Chang, Fuzzy topological spaces, Journal of Mathematical Analysis and Applications, 24, No. 1 (1968), 182-190.

[3] K. Atanassov, New operators defined over the intuitionistic fuzzy sets, Fuzzy Sets and Sysetms, 61, No. 2 (1993), 137-142.

[4] D. Çoker, An introduction to fuzzy subspaces in intuitionistic topological spaces, J. Fuzzy Math., 4 (1996), 749-764.

[5] D. Çoker, An introduction to intuitionistic fuzzy topological spaces, Fuzzy Sets and Systems, 88, No. 1 (1997), 81-89.

[6] S. K. Samanta and T. K. Mondal, On intuitionistic gradation of openness, Fuzzy Sets and Systems, 131, No. 3 (2002), 323-336.

[7] J. Gutiérrez Garcia and S. E. Rodabaugh, Order-theoretic, topological, categorical redundancies of interval-valued sets, grey sets, vague sets, interval-valued; intuitionistic sets, intuitionistic fuzzy sets and topologies, Fuzzy Sets and Systems, 156, No. 3 (2005), 445-484.

[8] Z.-G. Xu, H.-Y. Li and Z.-Q. Yun, Generalized fuzzy compactness in L-topological spaces, Bull. Malays. Math. Sci. Soc., 33, No. 2 (2010), 457-468.

[9] Kamal El-Saady, A. Ghareeb, Several types of $(r, s)$-fuzzy compactness defined by an $(r, s)$-fuzzy regular semiopen sets, Annals of Fuzzy Mathematics and Informatics, 3, No. 1 (2012), 159-169.

[10] E. p. Lee and Y. B. Im., Mated fuzzy topological spaces, Journal of fuzzy logic and intelligent systems, 11 (2001), 161-165.

[11] S.E. Abbas and E. El-Sanousy, Several types of double fuzzy semiclosed sets, The Journal of Fuzzy Mathematics, 20, No. 1 (2012), 89-102.

[12] A. M. Zahran, M. Azab Abd-Allah and A. Ghareeb, Several types of double fuzzy irresolute functions, International Journal of Computational Cognition, 8, No. 2 (2010), 19-23.

[13] R. Santhi and K. Arun Prakash, Intuitionistic fuzzy semi-generalized irresolute mappings, Tamkang Journal of Mathematics, 42, No. 2 (2011), 119-134.

[14] Fatimah. M. Mohammed, M. S. M. Noorani and A. Ghareeb, Slightly double fuzzy continuous functions, Journal of the Egyptian Mathematical Society, 23, No. 1 (2015), 173-179. 
